\title{
What can massively multiplayer online gaming do for urban public playgrounds?
}

\author{
J. Liu \\ Charles Sturt University, Australia
}

\begin{abstract}
Urban public playgrounds are key community hubs that support lifelong learning, healthy living, social integration, and civic engagement, but many of them are unable to fulfill their promises when emerging digital spaces such as online gaming and virtual reality challenge them. This paper takes a unique stance, envisaging digital spaces as opportunities rather than threats, and employs the uncertainty principle of massively multiplayer online gaming as a lens for examining urban public playground development. Drawing upon a critical analysis of a popular playground in Australia, it uncovers several key issues that might impede urban public playground development. It argues that future urban playground revitalization should not only address these issues but should also be proactive in facilitating inter-playspace communications, transitions, and connections.
\end{abstract}

Keywords: uncertainty, community development, play, interaction, play as learning.

\section{Introduction}

Urban communities worldwide are prioritizing public playground development as a future-oriented strategy for building sustainable communities that support lifelong learning, healthy living, social integration, and civic engagement. Many existing urban public playgrounds, however, are unable to fulfill their promises when emerging digital spaces such as social media, video gaming, and virtual reality, as well as the social and health problems resulting from the excessive use of these digital spaces, challenge them [1]. One major obstacle is a fixed, uniform view of play experience that fails to recognize the value of the digital habitus. Hence, this paper envisages digital spaces as opportunities rather than threats. It 
does so to seek insights from Internet gaming with the aim of revitalizing urban public playgrounds. The premise is that, since Internet gaming is rooted in play and is overwhelmingly popular, especially with young people, its virtual play mechanism should be translatable into play in physical space in one way or another. Therefore, the focus of attention is a particular genre of Internet gaming, massively multiple player online gaming, and its core principle, the uncertainty principle, as uncertainty is also a condition for undirected or free play [2]. It is believed that this change of focus can help engender new insights into urban public playspace cultivation as well as interdisciplinary alternatives.

\section{Urban public playgrounds and MMOG}

In many multicultural countries, such as the United States, Canada, and Australia, urban public playgrounds have become community hubs where learning, public health naturalization, social interaction, and civic engagement are intertwined. While the prevalent use of smart devices, social media, cloud computing, and wearable technologies makes playgrounds prominent learning spaces, it requires a different perspective of playgrounds. Some playgrounds, for instance, have installed electric carousels that children can control, while other playgrounds have attempted to install interactive whiteboards for digital doodling [3]. In a nutshell, these emerging functions demand the reconceptualization of public playground development. In overpopulated urban and metropolitan areas, where nature retreats and cement and the digital prevail, the demand is even more acute.

Over the years, three approaches for improving playground attraction and participation have emerged. The adventure playground approach is a nostalgic endeavor to restore the glory of adventure playgrounds, which were popular from the 1940s to the 1970s [4]. It encourages play autonomy, with few or no requirements for standardized play equipment. Recycled items such as used furniture, retired machinery parts, and farming tools can be repurposed to stimulate play. However, this is idealistic considering the associated demand for land and for play workers and the tightening of safety and health regulations.

The arts approach uses artwork to develop sustainable public playgrounds. It has been argued that adding aesthetic value to a playground improves playground participation and that artists should play a primary role in playground design and construction [5]. This approach is hardly sustainable as the success of a modern playground relies on the enhancement of aspects of play, such as interaction and challenges, and on the fulfillment of the playground's other functions.

The natural approach stresses the importance of using local or natural materials and landscapes for public playgrounds. This approach has strong potential as it regards playground development as physically situated and community-based [6]. However, it can be misleading as it positions globalization and digitization as threats to local cultures, ecologies, and employment rather than opportunities.

The recent rise of the inclusive and interactive approach may hold promise. This approach encourages participants of all abilities to engage in playground activities [7]. It also underscores the importance of interactions amongst participants and with devices [8]. Instead of excluding technologies, it encourages 
technology integration to create, say, narrative spaces in playgrounds. Unfortunately, to a large extent, it is still trapped in the deficit perception of digital spaces and is reluctant to further their exploration. For example, digitization through the Internet, cloud computing, virtual reality and augmented reality, modality profusion, and artificial intelligence is dissolving the boundaries between family, work, learning, and play while creating democratized access to resources and capital, enabling social actions, and deepening social connectivity.

Many innovations associated with digitization, such as apps, social networking, video streaming, and online gaming, can be exploited to imagine playground construction differently. The following questions come to mind. How can public playground attendance be increased? What new features can be added to playgrounds to enhance their sustainability as playspaces? How can playground participants share their play experiences? How can playground interaction be improved? Among the different digital avenues that could be explored, massively multiple online gaming is the most noteworthy for three main reasons. First, it exploits the play mechanism to such an extent that tens of millions of players are involved in it daily and many of them are developing various degrees of addiction [9]. It is not uncommon for a player to continue playing the same game, for instance, Warcraft or League of Legends, for years. The second reason for the significance of MMOG is that it emphasizes the value of teamwork and community to such an extent that players who exit in the middle of play may receive warnings and even punishments [10]. Third, it connects players across global and local communities, regardless of their sociocultural, ethnic, economic, and political backgrounds [11].

Disappointingly, over the years, the majority of research has centered on understanding MMOG as virtual space cultivation while discussing the translation of physical play ideas and activities into virtual play [12]. No research to reverse the trend of exploring MMOG's implications for physical playspace development has been attempted. The gamification hype may seem to have some relevance, but it is misplaced. It has been directed at reforming formal learning institutions, such as schools, that are disturbingly more interested in disciplining, control, conformity, and modelling [13]. Using gamification to refashion schooling in this regard is either a wasted investment or an aggravation of schooling's already miserable condition. Hence, MMOG's power should be directed towards other spaces (for instance, parks, playgrounds, beaches, and swimming pools), where informal, community-based learning resides, to be relevant and useful.

Many questions remain, including those concerning popularity or addiction, collaboration as being inclusive and communal, and connectivity that moves beyond inclusiveness and interaction. Some examples follow. What parts of the MMOG mechanism pertain to physical playspace construction? To what extent can an MMOG principle be applied in physical playspace planning? What constraints or obstacles might be expected? What are the potential issues, problems, or challenges that accompany application? What kinds of measures can be taken to safeguard or mitigate possible side effects? Apparently, the multiplicity and complexity of these issues require that emerging research focus on one of the 
issues as a starting point for the sake of feasibility. This paper's adoption of MMOG's uncertainty principle is one such attempt.

\section{The uncertainty principle of MMOG}

This study employs the uncertainty principle of MMOG as a framework for investigating urban public playgrounds. Costikyan $[14,15]$ argues that the uncertainty principle is one of the keys to successful online games. In his view, it encompasses five layers. As Figure 1 illustrates, the complexity layer stresses the necessity of creating a sufficiently complex system that gives balanced attention to all available actions. The randomness layer requires the dynamic generation of unexpected game states to challenge players with new situations and tasks, while outcomes are often unexpected. The motor execution layer concerns learning motor skills and fine-tuning the learned skills on a delicate scale or in response to the complexity of game states. The hidden information layer suggests that some information be deliberately concealed to make definite success impossible, even for advanced players. Unpredictability does not have to increase in line with complexity, but it does need to be consistently embedded to create surprises and even losses. The multiple player layer underscores the inevitability that players themselves can generate in complicating a game state, considering the variation amongst them in terms of ability and preferences. The greater the number of players involved, the more unpredictable gameplay can become.

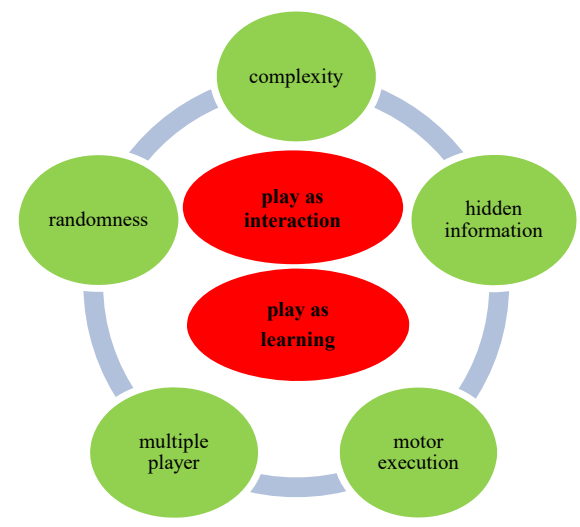

Figure 1: The uncertainty framework of MMOG.

These five layers of uncertainty revolve around two systems. One is based on the premise that any kind of gameplay is learning. In Fabricatore's [16] view, the manipulation of objects in play often requires a level of proficiency that is achievable through a learning process and the recognition of the objects' characteristics. In this sense, the play-as-learning premise transcends the simple vision of play as fun as well as the binary division between serious and recreational gaming. Games are varied in quality, but play itself is a process of learning, for 
learning, with learning, and through learning [17]. The acquired play skills, strategies, and competency are transferrable when game states are rightfully appropriated.

The other premise is that MMOG must be built on an imposed or mandatory interactive environment. In other words, gameplay cannot occur without interactions. In fact, its value often lies in the kinds of interactive frameworks that are built into the game system, whether they concern mapping, solving, evaluating, or understanding [18]. As Bedwell and Caruana [19] have pointed out, the spatial distribution of interactive zones, the level of the integration of devices, and content display often influence participant behaviors. Apparently, the adequate appropriation of the uncertainty framework requires the skillful, consistent exploration of play and interactive systems.

The main question that this analysis attempts to answer is: to what extent can the uncertainty framework of MMOG be used to understand urban public playground development? To answer this question, the study adopts the position that the public playground is a social and semiotic text. From this position, nonlinguistic means and modes, such as pictures, drawings, paintings, music, illustrations, and corporeal movements, can be utilized to create or negotiate meanings [20]. The present study also advocates for collecting data from online spaces, including websites, blogs, and YouTube, where various types of public playgrounds are curated.

\section{Darling Quarter Playground as a multimodal text}

Tumbalong Park, which is popularly known as Darling Quarter Playground, was chosen as the case for analysis. It is located at the very heart of the Sydney CBD and is immensely popular with local residents and tourists, whom it offers a balanced mix of exhibitions, performances, and events. The City of Sydney's website depicts the playground as an iconic public space for children and their families. It even describes children as "[t]he local area's most discerning critics." A website whose entire domain name is attributable to Darling Quarter praises the playground as "one of the most spectacular ever built in Australia, featuring an array of fun activities that promote learning and imagination for all ages." Both sites highlight water play as the playground's key feature.

ASPECT Studios, the landscape architecture team behind the planning of the entire Darling Quarter landscape, stresses in its summary that the project is "a transformation of the public domain of Darling Harbor South, one of Australia's most visited destinations" and that "with its interactive water play facilities [the playground] has become a regional attraction for Sydney." The playground was conceived of as a public space in which interactivity would play an essential role. Apparently, in its planning, ASPECT was acutely conscious of the positions of various stakeholders, including the Sydney Harbor Foreshore Authority (SHFA) and Lend Lease, and of the local community's strategic importance. That was evident in its statement: "[a] major 1.5-hectare place-making project for Sydney with a retail terrace, public park, two 6-star commercial buildings and an innovative children's playground as its center piece." In ASPECT's vision, the 
playground was the civic hub that connected social, cultural, and commercial activities in the area.

Kompan, a playground equipment manufacturer, designed and manufactured the play facilities and maintains them. In a promotional video that uses the Darling Quarter playground as a sample, Kompan describes the community as a commercial space for people to "sit, relax, and enjoy" and the playground as "integrating explorative play equipment and interactive water play in a landscaped environment." Regardless of the unconscious divide between children and adults, it takes pride in the central design principle that grafts exploration onto interactivity. Kompan's summary of the project states that "[t]he sensational, award winning water based playground in Sydney's Darling Quarter is a triumph in modern design" and that "after falling into disrepair, the former premises made way for a new, exciting redevelopment."

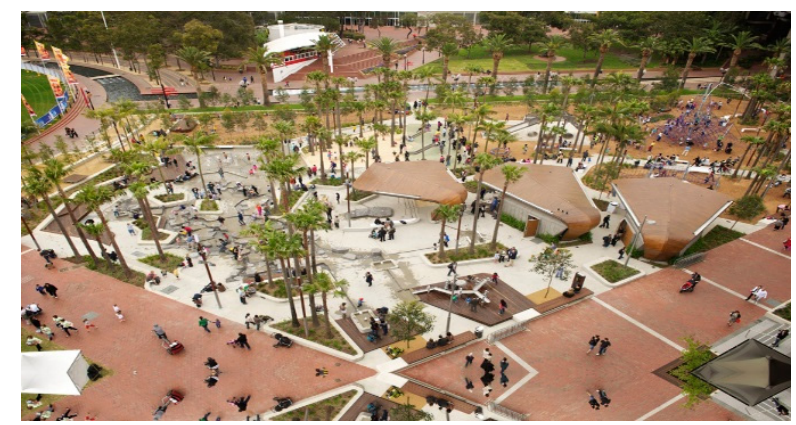

Figure 2: Darling Quarter Playground site.

As Figure 2 illustrates, the playground's typology includes the following play sections:

1. a water play section that consists of an LED fountain and waterways;

2. a sand play section that consists of an enclosed sandpit with two diggers and two small steel slides that enter the sandpit;

3. an equipment play section that consists of a wide slide built on a two-tunneled base (users need to climb up to the top of the slide using ropes or rock steps), a spinning ride, a spinning swing, two swings, a spider web for climbing, a flying fox for sliding, and walking ropes for balance exercises;

4. a kiosk section that consists of bathrooms, a parent's room, and a café; and

5. a rest section that consists of wooden decks and benches for caregivers and guardians.

Urbandesign, the official website of the Australian Sustainable Built Environment Council, uses the case of Darling Quarter playground to showcase the application of the twelve principles of Creating Places for People's protocol in the revitalization of Australia's urban spaces. However, it is not clear how these principles were integrated in the playground's development as it does not seem that Kompan used the protocol as a standard or a reference point. As is evident above, the water play section is the most captivating feature of the playground. 
Although the other sections have been promoted as innovative, they feature generic play equipment that is commonly installed in Australian urban public playgrounds. Therefore, the following analysis of uncertainty focuses solely on the water play activities. Table 1 below is a summary of the key play activities in each section of the water playground followed by detailed delineations.

Table 1: Water play activities.

\begin{tabular}{|l|l|l|}
\hline \multicolumn{1}{|c|}{$\begin{array}{c}\text { Play } \\
\text { section }\end{array}$} & \multicolumn{1}{|c|}{ Snapshot } & \multicolumn{1}{c|}{ Play activities } \\
\hline 1 & LED fountain & $\begin{array}{l}\text { Running around; sitting on water holes; } \\
\text { water splash; water fight; water dodging; } \\
\text { water dancing; water skidding; showering; } \\
\text { imaginative waterplay or role play. }\end{array}$ \\
\hline 2 & Water wheel & $\begin{array}{l}\text { Wheel spinning; wheel wrestling; water } \\
\text { splashing; water sliding (using paper boats, } \\
\text { coffee cups, water bottles, etc.); hide \& seek } \\
\text { (whirlpool underneath); water puddle } \\
\text { splashing; fishing; other imaginative play or } \\
\text { role play. }\end{array}$ \\
\hline 3 & Pump station & $\begin{array}{l}\text { Water pumping; water pumping race; water } \\
\text { collection and refilling (bottles, coffee cups, } \\
\text { plastic bags, balloons); other imaginative } \\
\text { play or role play. }\end{array}$ \\
\hline 4 & Water way & $\begin{array}{l}\text { Running/hopping around; water surfing; } \\
\text { waterway exploration; water-dam building; } \\
\text { water splashing; hide and seek (minimal); } \\
\text { paper boat rowing; fishing; other imaginative } \\
\text { play or role play. }\end{array}$ \\
\hline 5 & $\begin{array}{l}\text { Water pump spinning; water splashing; other } \\
\text { possible imaginative play or role play. }\end{array}$ \\
\hline
\end{tabular}

(Note: The play sections are hyperlinked to online images to save space.)

\section{Complexity}

Complexity is primarily enabled in the Water Way and Water Wheel sections. In the Water Way section, participants have multiple options: They can follow the multiple water ways to go upstream and downstream, open or close the mini-water gate, and skip or hop from one stream to another. Moreover, they can opt to play alone, in pairs, or in groups. In the Water Wheel section, varied volumes of water generate complexity: The faster the wheel spins, the greater the amount of water that flows into a water pipe and the whirlpool underneath it. It is common to see two or three younger children pushing and pulling together to spin the wheel while several other children splash water, fill bottles up, or row paper boats in the whirlpool. 


\section{Randomness}

Randomness is limited to water as a play medium and a space that participants can incorporate in their imaginative play. However, the play equipment does not allow or enhance randomness in any sense. Even in the LED Fountain section, participants are able to quickly work out the fountain's pattern and frequency, leaving very little room for surprise.

\section{Motor execution}

All water play sections may help in the development of various motor execution skills. However, only the Water Way and the LED Fountain sections cater to all participant motor skill development. Only certain age groups can use the Pump Station section, the Spinal Pump section, and the Water Wheel section. The LED Fountain section allows individualized performance, but, as a flat surface, it can neither create scaled motor-skill development nor provide varied motor execution skills.

\section{Hidden information}

This layer is absent in all the sections. However, it can be assumed that the engineering systems that support the LED Fountain and the Pump Station sections might intrigue some participants.

\section{Multiple players}

All sections but the Spinal Pump section allow multiple player interactions. The LED Fountain and the Water Way sections are accessible to players of all ages and genders as open playspaces. The Pump Station section is able to accommodate more than seven participants. The Water Wheel section requires two or more participants to move the wheel while four or five others collect water in the water tunnel and the whirl pool. Moreover, only one or two players can play in the Spinal Pump section, which is very heavily built with steel.

\section{Play as interaction}

Built as an open space that allows multiplayers in four of its five play sections, the water play space allows social exchanges among participants, including children of different ages and their caregivers. However, the majority of interactions occur with the play materials (namely, water) and equipment (such as the pump stations and water wheel). Furthermore, the majority of interactions are confined to close groups; they may include interactions with parents and guardians and with families, relatives, and friends. Interactions with strangers are limited. It is not clear whether various interactions with materials, equipment, participants, and places were explicitly planned in the playground design.

\section{Play as learning}

Participants in the water playground may be able to observe and learn social conventions without formal instruction or interventions, develop language and cultural awareness while immersed in a multilingual environment, and learn about materials and their attributes (water, stone, steel, cement; soft, cold, hard, dense; 
shapes, spaces, distances, color, temperature, density, motion, frequency, patterns; physical senses: fast, slow; pain; warm). However, it is not transparent how these kinds of skills were envisioned in the original water playground plan.

\section{Discussions and conclusion}

As is discussed above, the playground's stakeholders all believed that the award winning playground's popularity was built on its design excellence, which promoted play, particularly in the water play sections. However, the analysis of MMOG's uncertainty principle indicates that this may not be the case. The analysis reveals that the play materials and the open space facilitate four (namely, randomness, motor execution, complexity, multiplayers) of the five layers. The hidden information layer is not even activated. Play as learning occurs mainly on the surface; in other words, it is tied to the functions of equipment such as the water pump and the mini-dam gate. Such a design cannot invoke exploratory learning, which is connected to curiosity and creativity. Play as interaction, while confined to close groups, makes it difficult to create layered and networked social interactions among participants and with materials, resources, and places within and beyond the water playground.

In fact, such isolation of interaction is conspicuous when the water play section is designed as a standalone section rather than a section connected to other parts of the playground. It sits next to the sand play area, but, interestingly, the sandpit is walled off from the water play section with several warning signs that prohibit putting sand in the water. It is certainly a pragmatic measure, considering the cost of maintenance; yet the separation of water and sand reduces not only the complexity of play that two complex play materials afford but also interactions among participants. Ironically, many children and parents often ignore the warning signs - after all, not all of them are literate! Furthermore, play behaviors should not simply be regulated with warning signs; rather, thoughtful designs are advisable. The signs may, in fact, inflict a sense of authoritative intimidation and, consequently, non-belonging on the participants. In their place, some simple engineering mechanism that allows a smooth transition from the sand to the water can be devised to solve the problem.

It is known that the uncertainty principle's power lies in helping to generate virtual feedback loops in MMOG to sustain participation over a long period of time [21]. Such feedback loops can intensify participants' attention and desire to become loyal members of their gaming teams and communities. The above analysis makes it evident that the playground is unable to generate such desired feedback loops. Further, it raises the question of whether the playground's design has made any meaningful contribution to its immense popularity or other nondesign factors (such as the area's population density and its reputation as a worldrenowned tourist destination for domestic and international visitors) are the key contributors. If the latter is the case, it is plausible that any playspace with generic play equipment installed could become a catalyst for interactive play. The analysis raises another equally legitimate question: "What would happen if the playground were relocated to a different population-dense urban area?" It urges 
playground developers to rethink the criteria for assessing a public playground's success. In this case of Darling Quarter Playground, instead of boasting of aspects of their design philosophy (such as sustainability, the possibility of reducing "teenager-related issues and problems such as isolation and vandalism" and creating "positively influenced physical activity, social interaction and a sense of community ownership," and counting the numbers of visitors to the playground), the playground's developers should have invested more energy in examining, for example, how often play participants came to visit the playground, what their age or gender composition was, what play activities were ongoing, and in what manner or forms they existed.

In this respect, the present analysis of Sydney's most popular playground presents a worrisome picture of the growing gap between physical and virtual playgrounds' attraction of active participants. As the analyses have demonstrated, the uncertainty framework is inspiring for playground development and renovation. However, it should be developed further to make it more useful by working on characteristics such as scales, aspects, and categories. For instance, the hidden information layer should specify the place and the types and depth of the information that could be concealed. A further examination of the types of interactions suggested by Hespanhol and Tomitsch [22] is also necessary, namely, 1) performative interactions in which participants act in temporarily assumed roles, 2) embodied interactions in which participants become physically (and mentally) immersed in an environment, and 3) ubiquitous interactions in which participants establish a seamless process of playful communications with the surroundings. Without such elaboration, the framework would be of little use. Similarly, this preliminary one-case analysis is a stepping stone for further urban public playground research, which will involve multiple ethnographic case investigations across places (digital or physical) and cultures. Future urban playground development and revitalization should not only address these issues but should also be proactive in facilitating inter-playspace communication, transitions, and connections.

\section{References}

[1] Yao, K., Playground utilization: A study on urban, community and neighborhood park playgrounds in Manhattan, Kansas. (Masters dissertation), Kansas State University: Manhattan, Kansas, 2015.

[2] Waite, S., Huggins, V., Wickett, K., Maynard, T., \& Waters, J., Risky outdoor play: Embracing uncertainty in pursuit of learning (Chapter 5). Exploring Outdoor Play in the Early Years, eds T. Maynard and J. Waters, Open University Press, pp. 71-85, 2014.

[3] Young, J., \& Liu, A., Making sustainability visible: Two early childhood education centers. Paper presented at the ARCC Conference Repository, 2014.

[4] Gill, T., No fear: Growing up in a risk averse society. Calouste Gulbenkian Foundation: London, 2007. 
[5] Kingery-Page, K., \& Melvin, R. J., Site as experiential playground: Artistic research for a learning landscape. International Journal of Pedagogy and Curriculum, 19(4), pp. 71-89, 2013.

[6] Gemmell, J., Rethinking playgrounds: A design investigation of playscape theory. (Doctoral thesis), University of Maryland, College Park, 2015.

[7] Prellwitz, M., \& Skär, L., Usability of playgrounds for children with different abilities. Occupational therapy international, 14(3), pp. 144-155, 2007.

[8] Sturm, J., Bekker, T., Groenendaal, B., Wesselink, R., \& Eggen, B., Key issues for the successful design of an intelligent, interactive playground. Paper presented at the Proceedings of the 7 th International Conference on Interaction Design and Children, 2008.

[9] Blinka, L., \& Mikuška, J., The role of social motivation and sociability of gamers in online game addiction. Cyberpsychology: Journal of Psychosocial Research on Cyberspace, 8(2), 2014.

[10] Poor, N. D., Collaboration via cooperation and competition: Small community clustering in an MMO. Paper presented at the 47th Hawaii International Conference on System Sciences, 2014.

[11] Ahmed, D. T., \& Shirmohammadi, S., Networking for massively multiuser online gaming. Encyclopedia of Multimedia. Springer, pp. 664-670, 2008.

[12] Nosrati, M., Karimi, R., \& Hariri, M., General trends in multiplayer online games. World Applied Programming, 3(1), pp. 1-4, 2013.

[13] Mitra, S., The future of schooling: Children and learning at the edge of chaos. PROSPECTS, pp. 1-12, 2014.

[14] Costikyan, G., Uncertainty in games. MIT Press: Cambridge, MA, 2013.

[15] Costikyan, G., Sources of uncertainty: A bit of uncertainty in games. MIT Press: New York, 2014.

[16] Fabricatore, C., Learning and videogames: An unexploited synergy. Paper presented at the International Conference of the Association for Educational Communications and Technology, 2000.

[17] Mainemelis, C., Altman, Y., Kolb, A. Y., \& Kolb, D. A., Learning to play, playing to learn: A case study of a ludic learning space. Journal of Organizational Change Management, 23(1), pp. 26-50, 2010.

[18] Burgun, K. (2012). Game design theory: A new philosophy for understanding games. CRC Press, 2012.

[19] Bedwell, B., \& Caruana, T., Encouraging spectacle to create self-sustaining interactions at public displays. Paper presented at the Proceedings of the 2012 International Symposium on Pervasive Displays, 2012.

[20] Kress, G., Multimodality: A social semiotic approach to contemporary communication. Routledge: New York, 2013.

[21] Kuss, D. J., Internet gaming addiction: Current perspectives. Psychol Res Behav Manag, 6, pp. 125-137, 2013.

[22] Hespanhol, L., \& Tomitsch, M., Strategies for intuitive interaction in public urban spaces. Interacting with Computers, iwu051, 2105. 